\title{
Particle speciation analysis of inorganic polymer flocculants: an examination by photon correlation spectroscopy
}

\author{
Dongsheng Wang *, Hongxiao Tang, Fucang Cao
}

State Key Laboratory of Environmental Aquatic Chemistry, Research Center for Eco-Environmental Sciences, Chinese Academy of Sciences, POB 2871, Beijing 100085, People's Republic of China

Received 10 December 1998; accepted 3 September 1999

\begin{abstract}
The particle size distribution for two typical kinds of inorganic polymer flocculants is characterized comparatively by using photon correlation spectroscopy (PCS). The experimental results indicate that there exists large difference in particle speciation distribution between $\mathrm{PFCl}$ and $\mathrm{PACl}$ prepared under similar conditions. The particle size of $\mathrm{PFCl}$ samples range in a narrow distribution. Their mean effective hydrodynamic diameters calculated by translational diffusion coefficient are between 5 and $11 \mathrm{~nm}$. For PACl samples at certain basicity, no visible particle species can be determined. At high basicity, both laboratory prepared and commercial products, show a bimodal distribution: one in 2-5 $\mathrm{nm}$ and the other ranges in several decades of $\mathrm{nm}$ with wide distribution. The latter may be colloidal aluminum hydroxide and also some aggregates of polymers. (C) 2000 Elsevier Science B.V. All rights reserved.
\end{abstract}

Keywords: PCS; Inorganic polymer flocculant; Particle size distribution; Coagulation

\section{Introduction}

Inorganic polymer flocculants (IPFs) are a new kind of water and wastewater treatment reagents, which have been developed worldwide since the 1960 s [1,2]. There is a trend for them to become the main coagulant-flocculant because of their higher efficiency and relatively lower costs compared with the traditional coagulants. Presently they have been produced and used in a considerably large scale both in Japan, Russia, West Eu-

\footnotetext{
* Corresponding author. Fax: + 86-10-62923563.

E-mail address: wgds@mail.rcees.ac.cn (D. Wang)
}

rope and China [3]. The USA has also paid much attention to it in recent years $[4,5]$.

IPFs are the intermediate products of the hydrolysis-polymerization-ageing process of aluminum, iron and other salts under suitable conditions. There exist few established conclusions of the reason of their superior efficiency beyond the traditional coagulants. It is generally thought that the pre-produced products containing species of super quality and possess fairly stable structure against hydrolysis and solution chemistry, thus resulting in the higher coagulation efficiency [3]. However, the role of their physical property, especially the particle size distribution 
(PSD), remains unclear. It is believed that the PSD is of significant importance in the behaviour and efficiency of IPFs. While the speciation methods applied previously were based mainly on the chemical properties, little information on PSD has been obtained.

PCS, also referred to as quasi-elastic or dynamic light scattering (QELS or DLS), is routinely used to obtain particle size or size distribution information from the time-dependent fluctuations of scattered light intensity caused by concentration fluctuations (Brownian motion) of particles $[6,7]$. It has been widely used for particle size determination in the sub-micrometer range [8]. Since the pioneer work of Patterson et al., the application in aqueous chemistry of aluminum and iron and coagulation increases quickly [9-12]. While many other applications and modifications have also been investigated $[8,13,14]$.

The main purpose of this work is to characterize comparatively the particle species distribution of the typical two kinds of IPFs, i.e. PACl and $\mathrm{PFCl}$, by using PCS. In this preliminary study, we aim to get some important information on the physical-chemical features of IPFs and the nature of particle aggregation and growth process. Further work will then be concerned with coagulation dynamics and mechanisms [15].

\section{Materials and methods}

\subsection{Preparation of PFCl sample}

Stock standard solution of $2.3 \mathrm{~mol} \mathrm{1}^{-1}$ ferric chloride aqueous solution was prepared from analytical reagent grade $\mathrm{FeCl}_{3} \cdot 6 \mathrm{H}_{2} \mathrm{O}$ dissolved in distilled water. $\mathrm{PFCl}$ solutions were obtained by adding dropwise of increasing amounts of 0.5 mol $1^{-1} \mathrm{NaHCO}_{3}$ solutions under vigorous stirring at room temperature and at the appropriate concentration to obtain the desired basicity, $B$, from $0.2-2.4$. The final $\mathrm{Fe}$ concentration in the solutions is $0.058 \mathrm{~mol}^{-1}$. The same protocol was used to synthesize $0.23 \mathrm{~mol} 1^{-1} \mathrm{PFCl}$ solutions with the same values. All solutions were aged for $24 \mathrm{~h}$ for further PSD analysis.
Since the PCS determination can be greatly affected by impurities, stringent cleaning or purification practices were employed. As such, all glassware applied in subsequent PCS experiment was cleaned in the mixed cleaner and then copiously rinsed with doubly distilled water purified as described elsewhere [16]. Prior to PCS determination, $\mathrm{PFCl}$ samples were purified by filtering with $0.2 \mu \mathrm{m}$ Millipore membrane 4-6 times (depending on practical situation, the result can be checked by putting the sample on the laser beam and no bright spots should be observed coming from the passing beam). Then the purified samples were transferred into sample cell for further determination.

\subsection{Preparation of $\mathrm{PACl}$}

The stock solution of aluminum chloride was 1.0 mol $1^{-1}$, and similar method was used to prepare $\mathrm{PACl}$ solutions except some samples were titrated with $0.5 \mathrm{~mol} \mathrm{l}^{-1} \mathrm{NaOH}$ instead of $\mathrm{NaHCO}_{3}$. Commercial PACl solution (Joyful $\mathrm{PACl}$ ) was provided by Tangshan Factory, China.

\subsection{Instrument alignment}

The PCS measurements were performed on a Brookhaven Model BI200SM servomotor-controlled light scattering gonimeter with a Model BI9000AT autocorrelator (Brookhaven Instruments, Holtsville, NY). The incident light source was a $35 \mathrm{mw} \mathrm{He}-\mathrm{Ne}$ laser operating at a 632.8 $\mathrm{nm}$ wavelength with vertical polarization. The instrument alignment was checked by the BI-IST software, the deviations were less than $1 \%$ over the angular range.

\section{Results and discussions}

\subsection{Polystyrene latex standard}

During the measurement, it does not require any calibration, nevertheless it is appropriate to check its performance. For this reason, the instrument is first examined by using the standard 
latex particles. Here, we used the $96 \mathrm{~nm}$ latex (Nanosphere $^{\mathrm{TM}}$ Size Standards) supplied by the Duke Scientific Corporation (DSC). According to preparation method of literature [16], we measured the diameter of latex. The result of 8 times average is $99.3 \pm 2.2 \mathrm{~nm}$. Therefore the diameter obtained for the DSC sample is in excellent agreement with the stated value, $96 \pm 3.9 \mathrm{~nm}$.

\subsection{Particle size distribution (PSD) in PFCl}

The concentrations of samples of $\mathrm{PFCl}$ tested were $0.058 \mathrm{~mol}^{-1}$ and $0.23 \mathrm{~mol} \mathrm{l}^{-1}$, with $B$ value of $0.0,0.2,0.6,1.0,1.8,2.4$ respectively. During measurement, we found that the scattered intensity of samples with small $B$ value was too low to obtain reasonable results. The others of bigger $B$ value showed an intensity between 2 and $100 \mathrm{kcps}$. The samples appeared all to be a narrow distribution using the various software of the instrument. The average effective diameters calculated by cumulant method are shown in Table 1 .

The results show that the species in $\mathrm{PFCl}$ samples prepared by slow alkaline titration are gradually stabilized after $24 \mathrm{~h}$ ageing. The particulate species in $\mathrm{PFCl}$ are in a narrow distribution. There are little or no particle species in the low $B$ value samples and those existing in the higher $B$ value samples were of size between 5 and $11 \mathrm{~nm}$. Some individual ones are particular and exhibit wider distribution, especially when the $B$ value closes the region of first precipitation zone of $\mathrm{Fe}(\mathrm{OH})_{3}$, i.e. $B$ equals $0.4-0.5$. The sample concentration influences the PSD of $\mathrm{PFCl}$, as exemplified in the above experiments: the size increases a little with the low concentration. However, it may be caused by concentration effect during determination or results of both. Because any dilution of concentrated samples may result in redistribution of species, there remains some difficulty to make sure of the concentration effect during determination.

\section{3. $P S D$ in $P A C l$}

\subsubsection{Laboratory product}

Compared with $\mathrm{PFCl}$ of the same concentration, the samples of $\mathrm{PACl}$ show weaker scattering intensity. After $24 \mathrm{~h}$ ageing, the samples prepared by using $\mathrm{NaOH}$ at concentration of $0.05 \mathrm{~mol} \mathrm{1}^{-1}$ and various $B$ values are almost all undetectable for the faint scattering intensity. Only those of high $B$ value show some appearance at the initial ageing period, which exhibit an 'opalescent' phenomenon during sample preparation. But after several hours the solution returns to clear, the light intensity is then dropped to the noise level of the instrument accordingly. Increasing the sample concentration to $0.3 \mathrm{~mol}^{-1}$ and preparing with $\mathrm{NaHCO}_{3}$ at $B$ value of 2.0, the sample was monitored continuously during ageing. Fig. 1 is plotted as the average scattering intensity and particle size vs. time. It can be seen that during the initial ageing period, the scattering intensity increases slowly after preparation finished and then decreases after a maximum reached. This indicates that the polymers are still under slow aggregation and structure rearrangement after preparation. Accordingly, the particle size reaches a maximum and then decreases to a certain level. Fig. 2 is the typical result analyzed by NNLS after $24 \mathrm{~h}$ ageing. It shows a typical two sectional distribution. The small section is of size between 2 and $5 \mathrm{~nm}$, the large section are aggregates of size more than several decades of $\mathrm{nm}$ with a broad distribution.

\subsubsection{Commercial product}

Because there exists large difference between the process of laboratory preparation and industrial production, the composition and distribution

Table 1

The mean effective diameter of particle in $\mathrm{PFCl}$ sample (nm)

\begin{tabular}{|c|c|c|c|c|c|c|}
\hline$B(\mathrm{OH} / \mathrm{Fe})$ & 0.0 & 0.2 & 0.6 & 1.0 & 1.8 & 2.4 \\
\hline Concentration $0.058 \mathrm{~mol} \mathrm{l}^{-1}$ & $-{ }^{\mathrm{a}}$ & - & $24.1 \pm 0.5$ & $7.4 \pm 0.3$ & $6.3 \pm 0.5$ & $6.7 \pm 0.7$ \\
\hline Concentration $0.23 \mathrm{~mol} \mathrm{l}^{-1}$ & - & - & $10.5 \pm 0.6$ & $5.2 \pm 0.3$ & $5.1 \pm 0.5$ & $6.6 \pm 0.2$ \\
\hline
\end{tabular}

\footnotetext{
${ }^{\mathrm{a}}$ Without detectable particle.
} 


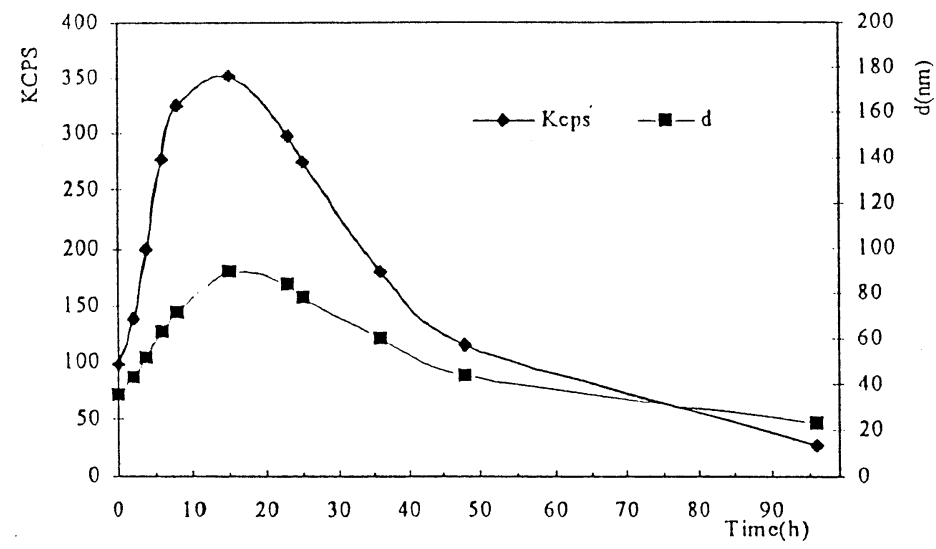

Fig. 1. The evolution of LS intensity and particle size of $\mathrm{PACl}$ with ageing.

of species may vary significantly. Generally speaking, the species of commercial products are relatively stable as indicated by the comparative study of Wang et al that the commercial solutions applied in industry resemble moderately aged laboratory OH-Al solutions [17]. The PSD of Joyful $\mathrm{PACl}$ is shown in Fig. 3: however, also a two sectional distribution.

\section{Discussion}

According to the lower limit of the instrument, what could be detected by PCS are particles of size over $1 \mathrm{~nm}$ or so. However, only that scattered intensity above $1 \mathrm{kcps}$ can trustworthy results be obtained. The effects of polydispersity fluctuations, minor 'dust' contamination, and hydrodynamic interactions are coexisting during determination of IPF samples, especially for $\mathrm{PACl}$ solutions, in which the scattering intensity is often too weak. However the above effects could be reduced by performing size determinations in the high-q region $\left(90-120^{\circ}\right)$. Anyway, whether the oligomer species of IPFs could be detected by PCS remains to be questioned.

Based on the basicity, $B$, or more exactly the hydrolysicity, $B^{*}$, Tang and Stumm classified $\mathrm{PFCl}$ solutions into four types [1]. Analyzed from the determination results, $\mathrm{PFCl}$ of type $\mathrm{A}$ and $\mathrm{B}$ do not exist detectable particle speciation. Only after a short period of induction (time period varies with $B$ values), the particles in type $B$ appear, exhibiting a rapid increase in scattering intensity, and precipitation is then observed. The particle size of type $\mathrm{C}$ is under some undulation, differ a little widely when $B$ value closes the first precipitation zone. As has been shown on the

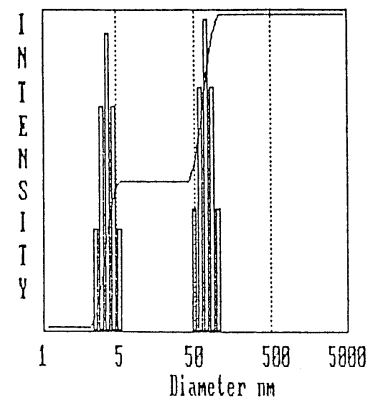

Fig. 2. The particle size distribution of laboratory prepared $\mathrm{PACl}$.

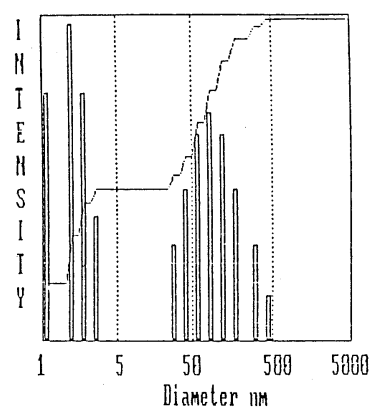

Fig. 3. The particle size distribution of commercial PACl. 
analysis of hydrolyzed $\mathrm{FeCl}_{3}-\mathrm{NaClO}_{4}$ system by Schwyn using small angle laser light scattering instrument [10]. The particle size of type D increases only a little according to their high basicity. Furthermore, the particle size decreases quickly in minutes with ageing, which could be easily monitored by PCS, indicating possibly that the low $\mathrm{pH}$ situation in $\mathrm{PFCl}$ samples causes the rapid species transformation. Therefore, the PCS results show clearly the different PSD in the four type $\mathrm{PFCl}$ solutions, and the $B^{*}$ is the deciding factor.

However, prepared under the same condition of PFCl, the PACI solutions show weaker scattering intensity. This indicates that the concentration or size of particles therein is too small to scatter enough light intensity. It appears that the particle speciation in PFCl and PACI prepared under the same condition vary significantly. In $\mathrm{PFCl}$ solutions solid iron oxyhydroxides, which are often in the size of several $\mathrm{nm}$, occur rapidly and readily on spending of polymers thus scatter stronger light. On the contrary, polymeric species in PACI are more stable and only in high $B$ value they may be aggregated into colloid aggregates, which exhibit a fractal structure as shown in the study of Bottero et al [18], and a wide PSD is often observed. During the initial ageing period, the aggregates undergo a slower transformation after preparation as shown in Fig. 3 and differ notably with $\mathrm{PFCl}$. The results of this analysis present a direct verification of the different speciation distribution character in PACI and $\mathrm{PFCl}$ and can thus shed some light on the interpretation on the difference of the coagulation mechanism between Fe-based and Al-based coagulants. It needs to point out that since the particle speciation existing in IPF is of the range of nanometer, to increase the laser power may yield more information.

\section{Conclusion}

Preliminary results reported herein that PCS is capable of providing valuable size information of IPF and dynamic transformation properties of species during ageing. The results show that there exists remarkable difference of PSD between $\mathrm{PFCl}$ and $\mathrm{PACl}$, therefore may influence their subsequent coagulation effect. The particle size of $\mathrm{PFCl}$ samples range in a narrow distribution. Increase the $B$ values of samples, only increase a little the size of particles. While in PACl samples, both laboratory prepared and commercial product, show a typical bimodal distribution at certain $B$ value. During initial ageing, the aggregates undergo slow transformation after preparation for $\mathrm{PACl}$ and differ notably with $\mathrm{PFCl}$.

\section{Acknowledgements}

Professor Weiner and Mrs. Helen of BIC Company are greatly acknowledged for their help on the instrument alignment. During the revision of this paper, the kind suggestions from Professor Gregory of UCL, UK and also two anonymous reviewers are appreciated. This work is supported by the NSF of China (29807004 and 59778019).

\section{References}

[1] H.X. Tang, W. Stumm, The coagulation behavior of Fe(III) polymeric species-I, Water Res. 21 (1) (1987) 115.

[2] H.X. Tang, Z.K. Luan, D.S. Wang, B.Y. Gao, Composite inorganic polymer flocculant, in: H.H Hahn, E. Hoffman, H. Odegaard (Eds.), Chemical Water and Wastewater Treatment V, Springer, 1998.

[3] H.X. Tang, The basic research of inorganic polymer flocculant, Environ. Chem. 9 (3) (1990) 1-12 (in Chinese).

[4] AWWA coagulation committee, Committee report: coagulation as integrated water treatment process, 81 (10) (1989) $72-78$.

[5] S.K. Dentel, Coagulation control in water treatment, CRC Crit. Rev. Environ. Control 21 (1991) 41-135.

[6] H.G. Barth, S.T. Sun, Particle size analysis, Anal. Chem. 67 (12) (1995) 257R-272R.

[7] B.B. Weiner, W.W. Tscharnuter, in: T. Provder (Ed.) ACS Symposium Series 332, ACS, W.D.C., (1987) 48.

[8] G. Bryant, C. Abeynayake, J.C. Thomas, Improved particle size distribution measurements using multiangle dynamic light scattering. 2. Refinements and applications, Langmuir 12 (1996) 6224-6228.

[9] J.H. Patterson, J.R. Tyree, A light scattering study of the hydrolytic polymerization of aluminun, J. Colloid Interf. Sci. 43 (2) (1973) 389-398.

[10] B. Schwyn, Ph.D. Dissertation, ETH, Switzerland, (1984). 
[11] J.Y. Bottero, Prog. Wat. Tech. 12 (1980) 601-612.

[12] J.Y. Bottero, D. Tchoubar, M. Arnaud, Partial hydrolysis of ferric chloride salt, Langmuir 7 (5) (1991) 13651369.

[13] J.H.V. Zanten, M. Elimelech, Determination of absolute coagulation rate constants by multiangle light scattering, J. Colloid Interf. Sci. 149 (1992) 528.

[14] V.A. Hackley, M. Anderson, Effects of short-range forces on the long-range structure of hydrous iron oxide aggregates, Langmuir 5 (1) (1989) 191.

[15] Wang, D.S., Tang, H.X., Gregory, J., Role of specia- tion on coagulation of kaolin with inorganic polymer flocculant: a dynamic investigation by turbidity fluctuations monitoring, (in preparation), (1999).

[16] BIC instrument manual for BI200SM and BI9000AT

[17] W.Z. Wang, P.H. Shu, The nature of polynuclear $\mathrm{OH}-$ Al complexes in laboratory-hydrolyzed and commercial hydroxyl aluminum solution, Clays Clay Minerals 42 (3) (1994) 356-368.

[18] J.Y. Bottero, M. Axelos, D. Tchoubar, Mechanism of formation of aluminum trioxide from keggin $\mathrm{Al}_{13}$ polymer, J. Colloid Interf. Sci. 117 (1987) 47. 